\title{
Simulations of Mini-EUSO observations of UV phenomena in the atmosphere
}

\author{
G. Suino*, M. Bertaina, E. Bertola, R. Bonino, F. Fenu, A. Liberatore ${ }^{\dagger}$ \\ Department of Physics, University of Torino \& INFN Torino - Italy \\ E-mail: gregoriosuino@gmail.com
}

\section{A. Cellino}

Istituto Nazionale di Astrofisica OATo - Italy

\section{Conti, M. Perdichizzi, A. Nardelli}

Uninettuno University, Rome \& INFN Roma Tor Vergata - Italy

\section{F. Capel}

Royal Institute of Technology, Stockholm - Sweden

\section{Piotrowski}

RIKEN - Japan

\section{for the JEM-EUSO Collaboration}

\begin{abstract}
The Mini-EUSO instrument is an UV telescope to be placed aboard the International Space Station (ISS) in the framework of the JEM-EUSO project. Mini-EUSO will map the Earth in the UV range $(300-400 \mathrm{~nm}$ ) with a spatial resolution of $5 \mathrm{~km}$ and a temporal resolution of $2.5 \mu \mathrm{s}$. Therefore, Mini-EUSO offers an excellent opportunity to study a variety of physics phenomena such as transient luminous events and meteors, as well as searching for strange quark matter and test the detection of space debris. Mini-EUSO will serve also as a pathfinder for the study of Extreme Energy Cosmic Rays from space. A review on the expected performance of Mini-EUSO in detecting a variety of physical phenomena simulated with the EUSO Simulation and Analysis Framework (ESAF) package is described.
\end{abstract}

35th International Cosmic Ray Conference - ICRC2017

10-20 July, 2017

Bexco, Busan, Korea

\footnotetext{
* Speaker.

$\dagger$ This work is dedicated to the memory of Yoshiya Kawasaki and Jacek Karczmarczyk, members of the JEM-EUSO Collaboration, passed away in 2016.
} 


\section{Introduction}

Mini-EUSO [1, 2] is a scientific mission within the JEM-EUSO program [3]. The main goal of Mini-EUSO is to measure the UV emissions from the ground and atmosphere, using an orbital platform. These observations will provide interesting data for the scientific study of a variety of UV phenomena such as transient luminous events (TLEs), meteors, space debris, hypothetical strange quark matter (SQM) and bioluminescence [4], as summarised in Fig. 1. Moreover, this will allow us to characterise the UV emission level, which is essential for the optimisation of the design of future EUSO instruments for Extreme-Energy Cosmic Ray (EECR) detection. Mini-EUSO will observe

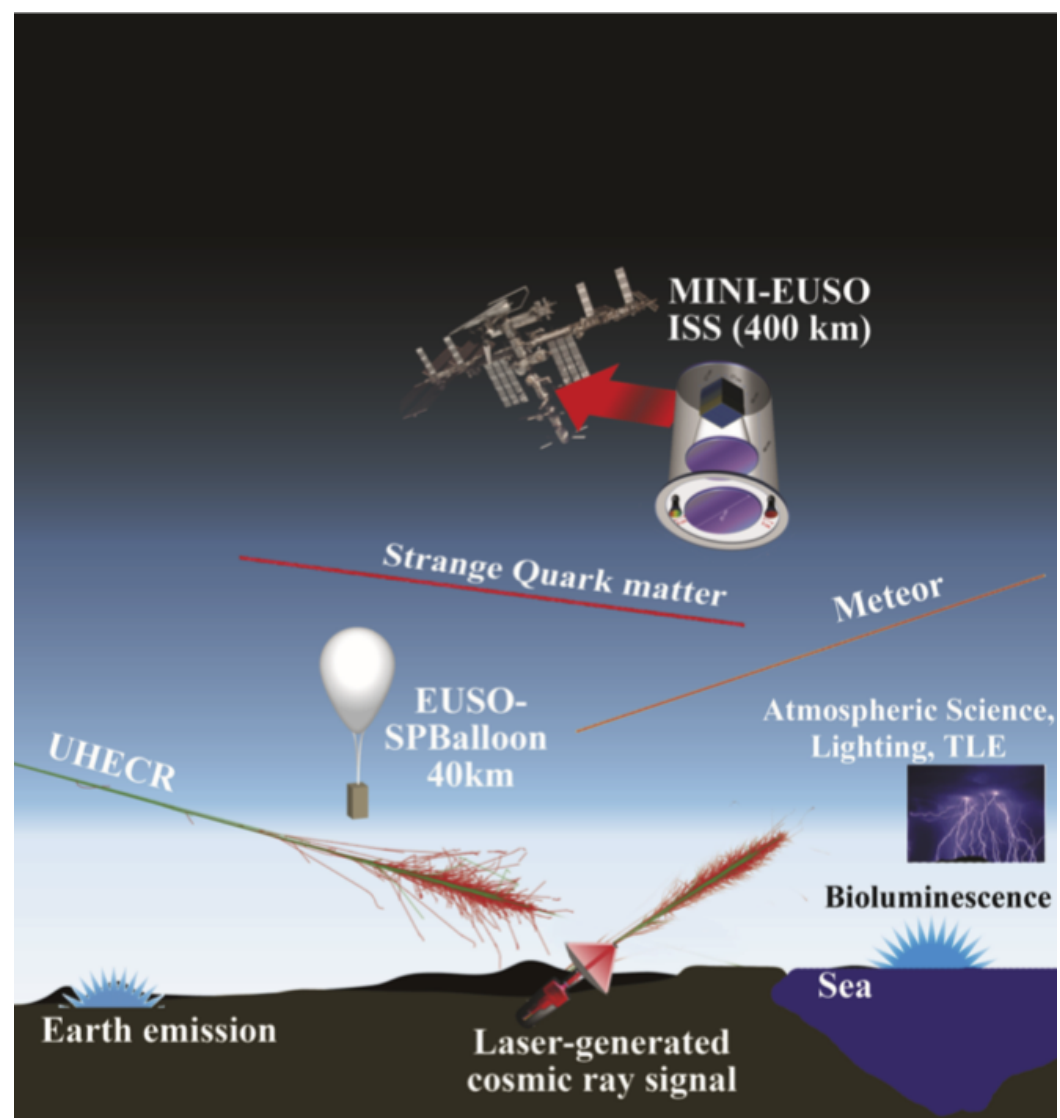

Figure 1: The Mini-EUSO mission summarised in one diagram. From the ISS, Mini-EUSO will observe a variety of interesting phenomena in the UV range, in addition to creating a high resolution UV map of the Earth.

the atmosphere from a nadir-facing window inside the Zvezda module of the ISS. It is based on one EUSO detection unit, referred to as the Photo Detector Module (PDM). The PDM consists of 36 multi-anode photomultiplier tubes (MAPMTs), each one having 64 pixels, for a total of 2304 pixels. The MAPMTs are provided by Hamamatsu Photonics, model R11265-M64, and are covered with a $2 \mathrm{~mm}$ BG3 UV filter with anti-reflective coating. The full Mini-EUSO telescope includes 3 main systems: the optical system; the PDM; and the data acquisition system [5]. The optical system of 2 Fresnel lenses is used to focus light onto the PDM in order to achieve a large Field of view $\left(44^{\circ} \times 44^{\circ}\right)$ with a relatively light and compact design, well-suited for space application [6]. The 
PDM detects UV photons and is read out by the data acquisition system with a sampling rate of $2.5 \mu$ s and a spatial resolution of $6 \mathrm{~km}$. The results described here represent a subset of those summarised in [1].

\section{Simulations of typical observations}

The Mini-EUSO configuration has been included in the ESAF (EUSO Simulation and Analysis Software) package [7]. ESAF is one of the official software tools to perform simulations of Extensive Air Shower (EAS) development, photon production and transport through the atmosphere and detector response for optics and electronics. Moreover, ESAF includes algorithms for the reconstruction of the properties of EAS produced by EECRs. Originally developed for the ESA-EUSO mission, all the planned missions of the JEM-EUSO program have been implemented in ESAF in order to assess the full range of expected performance for cosmic ray observation. In parallel, efforts are currently being pursued to implement the Mini-EUSO configuration in the Offline code [8] which is the other official software framework of the JEM-EUSO collaboration.

Although Mini-EUSO is not designed to detect EECRs due to the small size of the optical system, it will be possible to provide an upper limit for a null detection with its large annual exposure of $15000 \mathrm{~km}^{2}$ sr above $10^{21} \mathrm{eV}$ (see Fig. 2). A UV background level of 1 photon/pixel/GTU was considered which correspond to a UV nightglow intensity of $\sim 500$ photons $/ \mathrm{m}^{2} / \mathrm{ns} / \mathrm{sr}$, the typical value expected on oceans during dark nights [9]. Additionally, during flight it will be possible

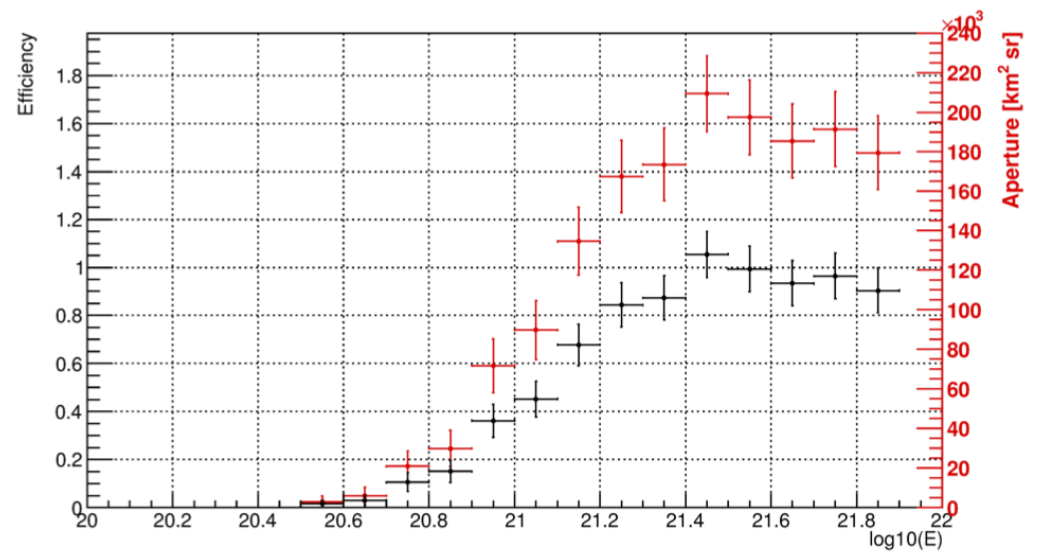

Figure 2: The detection exposure efficiency (on the left axis, in black) and geometrical aperture (on the right axis, in red) are shown as a function of the EAS energy, E, in eV. A UV background level of 1 photon/pixel/GTU was considered in both cases.

to simulate EECR-like signals using ground-based laser facilities in order to verify the capability of Mini-EUSO to detect cosmic rays and to allow the testing and optimisation of the trigger system [10].

TLEs such as blue jets, sprites and elves have been discovered relatively recently and are still not well understood [11]. They have typical durations of tens of ms. These upper-atmospheric events have UV luminosity and high frequencies [12], and thus should be well characterised to avoid interference with EECR detection and triggering. Mini-EUSO has a dedicated trigger algorithm to capture TLEs and other millisecond scale phenomena at high resolution [10]. These data 
could help improve the understanding of the formation mechanisms of filamentary plasma structures, complementing atmospheric science experiments. Fig. 3 shows examples of typical TLEs as they are simulated in Mini-EUSO. The simulations employ Toy Models which in a simplified way reproduce the size, shape and wavelength spectra of the different physical phenomena. Details of the implementation of these phenomena in ESAF can be found in [13]. The HV switching system of Mini-EUSO will modify the detection efficiency of the MAPMTs by changing the voltage between cathode and the first dynodes. In this way, the full dynamic range of Mini-EUSO spans over 6 orders of magnitude in photon flux and many different types of TLEs can be detected.
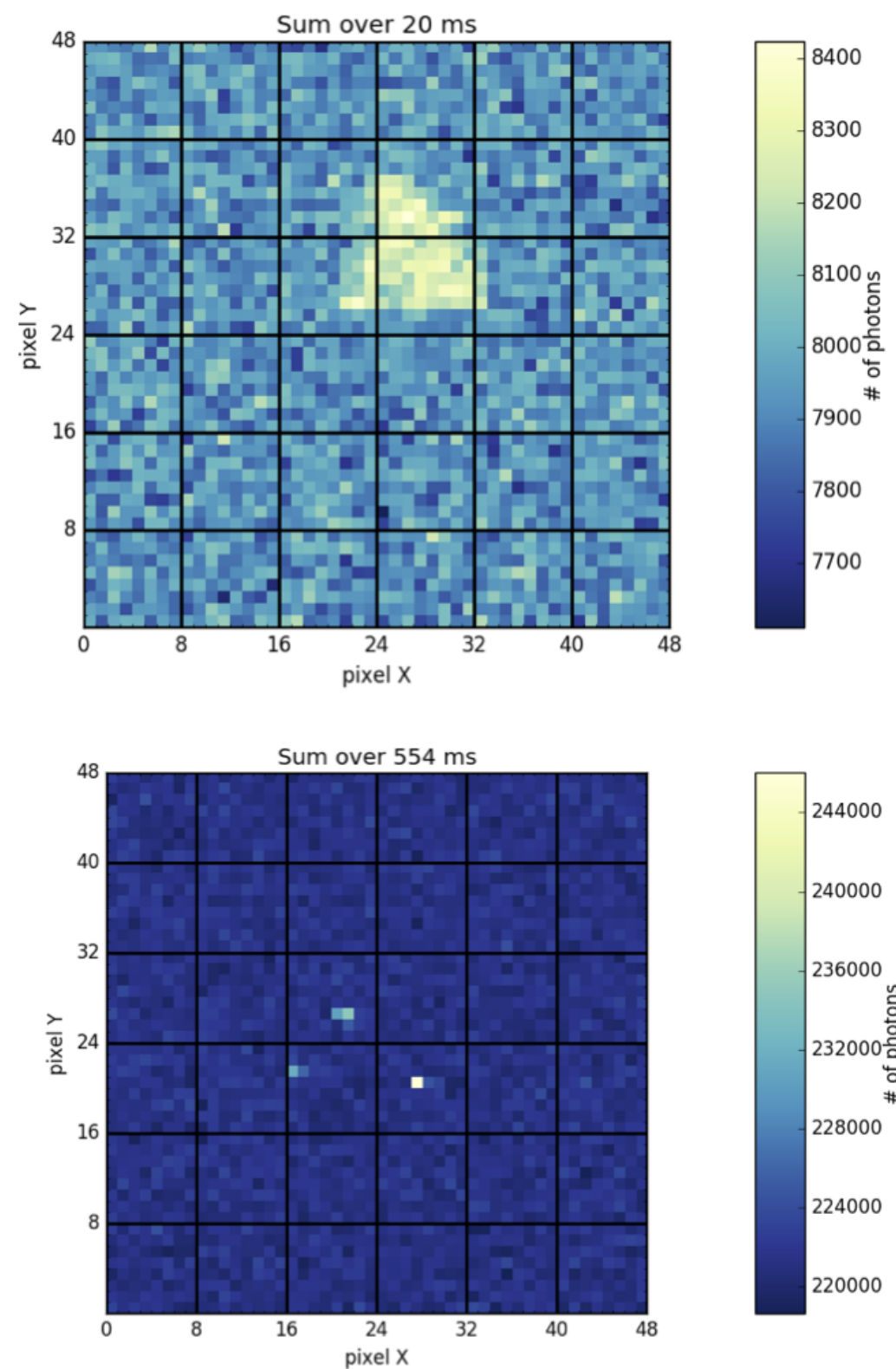

Figure 3: Expected light track of a diffuse elve (top) and 3 localised blue jet events (bottom) as they would be detected by Mini-EUSO. Background emission of 1 photon/pixel/GTU is also included. 
Mini-EUSO will also be able to detect slower events such as meteors, fireballs, strange quark matter (SQM) and space debris with magnitudes of $\mathrm{M}<+5$. In optimal dark conditions, the signal (integrated at steps of $40 \mathrm{~ms}$ ) will exceed the UV-nightglow level by 3-4 $\sigma$. These events will be detected using offline trigger algorithms on ground. The implementation of the meteor phenomena in ESAF is described in [14], which inherits the approach described in [15].

Fig. 4 shows an example of a meteor track having absolute magnitude $\mathrm{M}=+5$ crossing the field of view of Mini-EUSO with a $45^{\circ}$ inclination with respect to the nadir axis. The meteor speed is $70 \mathrm{~km} \mathrm{~s}^{-1}$ and its duration is $2 \mathrm{~s}$.

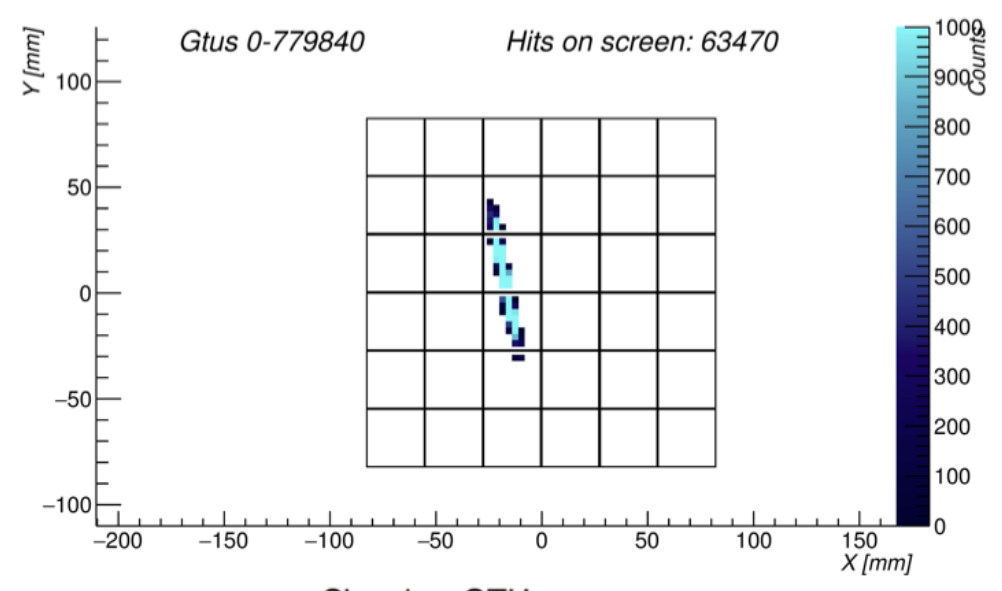

Signal vs GTU

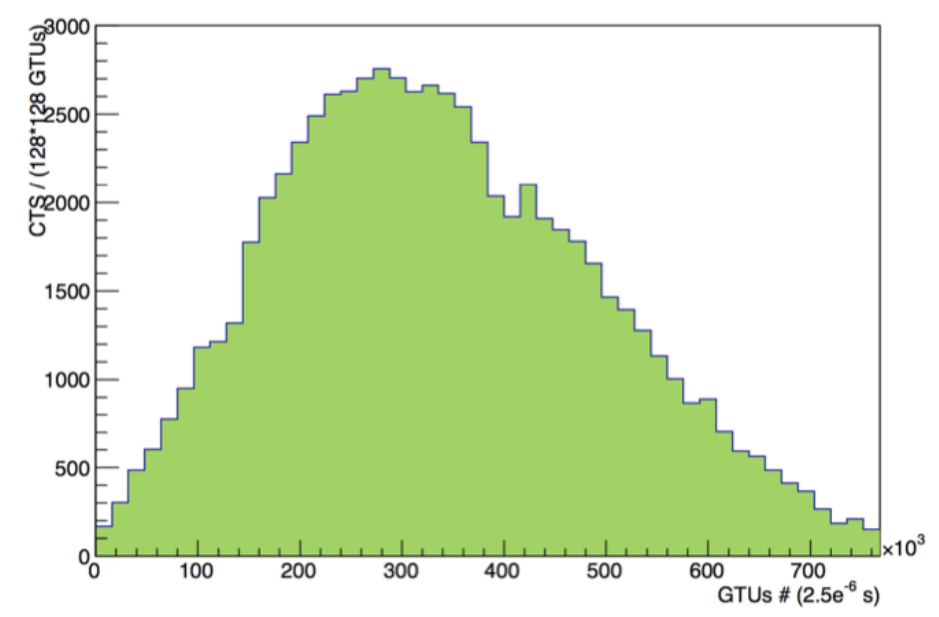

Figure 4: Expected light track of a meteor of absolute magnitude $M=+5$ detected by Mini-EUSO (the effects of UV-nightglow are not included and a threshold has been applied at 30 counts). Bottom: Expected light profile. Each time bin on the x-axis corresponds to an integration time of $40.96 \mathrm{~ms}$, the resolution of the level 3 data from Mini-EUSO.

Even after just 1 month of observation at a minimal UV-nightglow level, Mini-EUSO will be able to set a new upper limit on the detection of SQM, as shown in Fig. 5. SQM is composed of roughly equal numbers of up, down and strange quarks, and can form stable macroscopic nuggets referred to as nuclearites. As described in [15], these nuclearites create a UV signal upon inter- 
action with the atmosphere which can be detected by Mini-EUSO. The nuclearite signal is easily discerned from meteor tracks as we expect velocities much higher than $\sim 100 \mathrm{~km} \mathrm{~s}^{-1}$, compared to a maximum of around $72 \mathrm{~km} \mathrm{~s}^{-1}$ for meteors. The fig. 5 shows the upper limit on the nuclearite flux of $10^{-21} \mathrm{~cm}^{-2} \mathrm{sr}^{-1} \mathrm{~s}^{-1}$ for a null detection of nuclearites based on the conservative assumption that events with a velocity below $190 \mathrm{~km} \mathrm{~s}^{-1}$ are rejected.

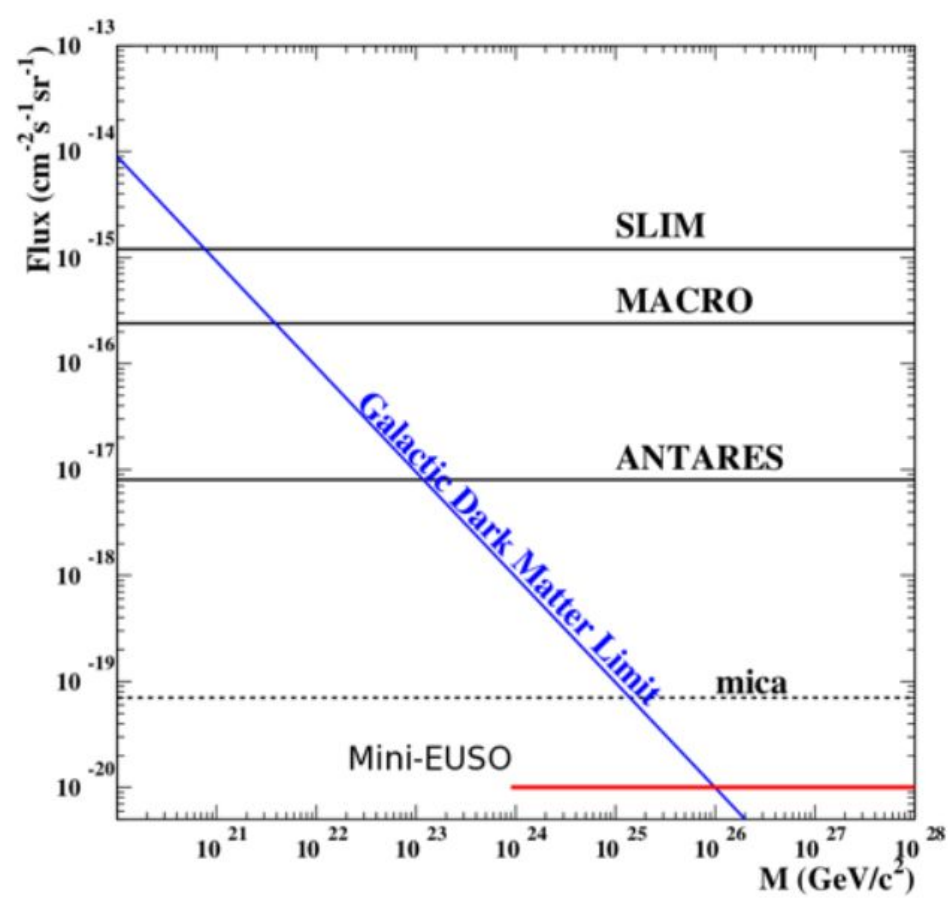

Figure 5: Upper limit to SQM with Mini-EUSO after $24 \mathrm{~h}$ observation as compared to current data [16, 17, $18,19]$.

The observation of space debris is also a highly relevant issue. Mini-EUSO is effectively a high-speed camera with a large field of view and will be used as a prototype for the detection of space debris during the twilight periods of observation. It will detect debris when they are illuminated by the Sun, but the instrument is in darkness. It is clear that a camera operating in the visible range would be more effective, though the Sun is quite bright also in the $300-400 \mathrm{~nm}$ range. In the current simulation within ESAF the photon flux of the Sun in the $300-400 \mathrm{~nm}$ has been considered. The debris are assumed to have a spherical shape of diameter $d$ and a variable reflectance. The fig. 5 shows the potential of Mini-EUSO to detect space debris. The condition set in the simulation is that the signal is at least $4 \sigma$ above background for at least 5 consecutive blocks of $40.96 \mathrm{~ms}$ each. The UV background has been assumed to be the same as in the other simulations which is 1 count/pixel/GTU. However, it is possible that for this specific measurement the background could be higher due to the presence of some sunlight, therefore, more precise calculation of the effective UV intensity will be performed in future. 


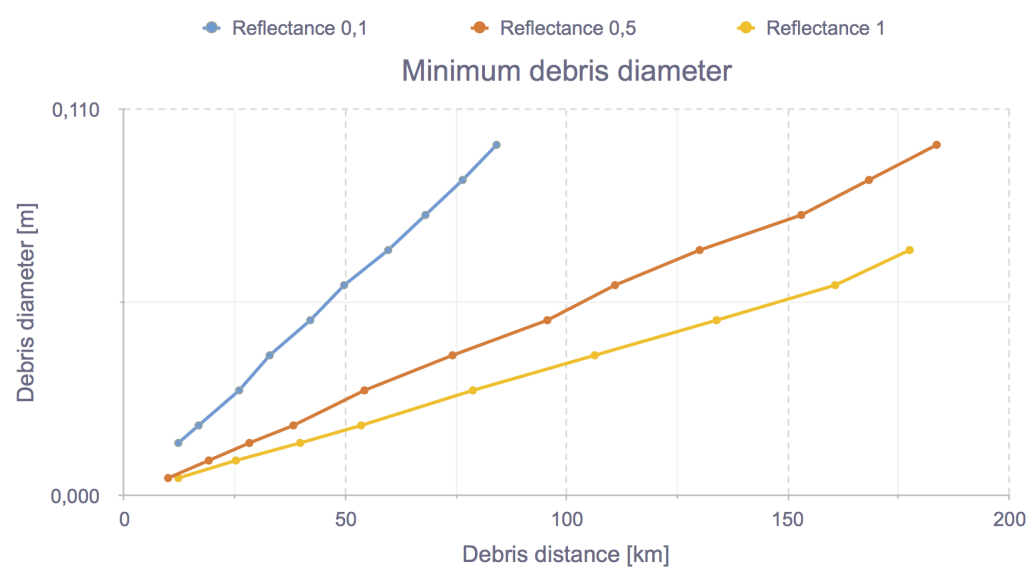

Figure 6: Minimum size of space debris observable by Mini-EUSO as a function of reflectance and distance from the International Space Station.

\section{Conclusions}

In summary, Mini-EUSO is a compact UV telescope that will observe from a nadir-facing window inside the Zvezda module of the ISS. The instrument employs a multi-level trigger system. Mini-EUSO will provide insight into a variety of atmospheric and terrestrial UV phenomena (with complementary information from the NIR and visible light cameras) as well as raising the technology readiness level of future EUSO missions. Typical observations have been simulated in order to verify the achievements of the mission goals. Mini-EUSO is approved as a joint project by the Italian (ASI) and Russian (Roscosmos) space agencies and the instrument integration is currently at an advanced stage to be on schedule with a possible launch in late 2017 to early 2018 .

\section{Ackwowledgments}

This work was partially supported by NASA award 11-APRA-0058 in the USA, by Basic Science Interdisciplinary Research Projects of RIKEN and JSPS KAKENHI Grant (22340063, 23340081, and 24244042), by the Italian Ministry of Foreign Affairs and International Cooperation, by the Italian Space Agency ASI contract 2016-1-U.0 "Mini-EUSO, by the 'Helmholtz Alliance for Astroparticle Physics HAP' funded by the Initiative and Networking Fund of the Helmholtz Association, Germany, and by Slovak Academy of Sciences MVTS JEMEUSO as well as VEGA grant agency project 2/0076/13. Russia is supported by the Russian Foundation for Basic Research Grant No 13-02-12175-ofi-m, Grant No 15-35-21038-mol-a-ved, Grant No 16-29-13065. The Spanish Consortium involved in the JEM-EUSO Space Mission is funded by MICINN \& MINECO under the Space Program projects: AYA2009- 06037-E/AYA, AYA-ESP2010-19082, AYA-ESP201129489-C03, AYA-ESP2012-39115-C03, AYA-ESP2013-47816- C4, MINECO/FEDER-UNAH134E-2741, CSD2009-00064 (Consolider MULTIDARK) and by Comunidad de Madrid (CAM) under projects S2009/ESP-1496 \& S2013/ICE-2822. 


\section{References}

[1] F. Capel at al. (JEM-EUSO Coll.), submitted to Advances in Space Research (2017).

[2] M. Ricci at al. (JEM-EUSO Coll.), "Mini-EUSO: a precursor mission to observe and study Atmosphere and Earth UV emission from the International Space Station", This Conference Proceedings, Busan (2017).

[3] J. Adams at al. (JEM-EUSO Coll.), Experimental Astronomy, Vol. 40(1), p.1 (2015).

[4] M. Casolino at al. (JEM-EUSO Coll.), "Science of MINI-EUSO experiment on the International Space Station", This Conference Proceedings, Busan (2017).

[5] A. Belov at al. (JEM-EUSO Coll.), "Mini-EUSO photodetector module data processing system", This Conference Proceedings, Busan (2017).

[6] Y. Takizawa at al. (JEM-EUSO Coll.), "Mini-EUSO optics design and its optical measurements", This Conference Proceedings, Busan (2017).

[7] M. Bertaina at al. (JEM-EUSO Coll.), Advances in Space Research, Vol. 53(10), p.1535 (2014).

[8] T. Paul at al. (JEM-EUSO Coll.), "New simulation and reconstruction software for the EUSO pathfinders, with example applications", This Conference Proceedings, Busan (2017).

[9] J.H. Adams at al. (JEM-EUSO Coll.), Astroparticle Physics, Vol. 44, p.76 (2013).

[10] F. Capel at al. (JEM-EUSO Coll.), "The Mini-EUSO multilevel trigger algorithm and its performance", This Conference Proceedings, Busan (2017).

[11] V. P. Pasko et al., Space Sciences Review, 168(1-4), 475 (2011).

[12] G. K. Garipov et al., Astroparticle Physics, 24(4-5), 400 (2005).

[13] M. Perdichizzi, Simulation of TLE phenomena in ESAF within the JEM-EUSO project, Bachelor Thesis, Uninettuno University (2015).

[14] A. Nardelli, Meteor simulation in ESAF for the JEM-EUSO mission, Bachelor Thesis, Uninettuno University (2014).

[15] J. Adams et al. (JEM-EUSO Coll.), Experimental Astronomy, Vol. 40(1), p.253 (2015).

[16] Ambrosio, M., et al. (MACRO Coll.), Eur. Phys. J. C. 13, p.453 (2000).

[17] Cecchini, S., et al. (SLIM Coll.), Eur. Phys. J. C. 57, p.525 (2008).

[18] Pavalas, G.E., et al. (ANTARES Coll.), Proceedings of the 23rd European Cosmic Ray Symposium, Moscow, 543 (2012).

[19] Price, P.B., Phys. Rev. D 38, p.3813 (1988). 